\title{
Computational Intelligent Design of 2D Nanostructures Based on Carbon
}

\author{
T. Burczyński1 ${ }^{*}$, A. Mrozek ${ }^{2}$, W. Kuśs \\ ${ }^{1}$ Institute of Fundamental Technological Research, Polish Academy of Sciences, Pawinskiego \\ 5B, 02-106 Warsaw, Poland \\ e-mail: tburczyński@ippt.pan.pl \\ ${ }^{2}$ AGH University of Sciences and Technology, Kraków, Poland \\ e-mail: amrozek@agh.edu.pl \\ ${ }^{3}$ Silesian University of Technology, Gliwice, Poland \\ e-mail: waclaw.kus@polsl.pl \\ *corresponding author
}

\begin{abstract}
The paper describes an application of the hybrid intelligent algorithm to optimal searching for new, stable atomic arrangements of 2D graphene-like carbon lattices. The proposed approach combines the parallel evolutionary algorithm and the conjugated-gradient optimization technique. The main goal is to find stable arrangements of carbon atoms under certain imposed conditions such as density, shape and size of the unit cell and also predefined mechanical properties. The nanostructure is considered a discrete atomic model and interactions between atoms are modeled using the AIREBO potential, especially developed for carbon. The parallel approach is used in computations. Validation of the obtained results and examples of new models of the new grapheme-like materials are presented.
\end{abstract}

Keywords: Hybrid intelligent algorithm, 2D nanostructures, new grapheme-like materials

\section{Introduction}

Carbon atoms form various types of bondings and spatial configurations. This ability is determined by the atoms' hybridization states, which depend on their particular electronic configuration. This phenomenon is responsible for the existence of many different allotropes of the carbon. This is due to unique electronic, thermal and mechanical properties of such structures. Additionally, 2D graphene-like materials can be used to create another, more complex class of nanostructures, such as nanotubes. Graphene-like materials can be classified as periodic, flat atomic networks, made of stable configurations of carbon atoms in certain hybridization states. Since the stable configurations of atoms correspond to the global minima on the Potential Energy Surface (PES), such a task can be considered a special optimization problem in which optimal material layout is searched on the nano-scale. However, the number of local minima increases almost exponentially with the number of atoms in the considered structure, thus searching for the global minimum on a PES became a non-trivial, NP-hard problem. 


\section{Hybrid intelligent algorithm}

Hybrid The hybrid algorithm, proposed and presented in this paper, combines the parallel Evolutionary Algorithm (EA) prepared by the authors, and the classical Conjugated-Gradient (CG) minimization of the total potential energy of the optimized atomic system. Since the processed structure is considered a discrete atomic model, the behavior and the potential energy of carbon atoms are determined using the Adaptive Intermolecular Reactive Empirical Bond Order (AIREBO) (Stuart et al. 2000) potential developed for molecular dynamics simulations of hydrocarbons.

A chromosome represents design variables in the form of real-valued Cartesian coordinates of each atom in the considered unit cell of the newly created atomic lattice. Each chromosome represents a certain spatial arrangement of atoms. In the initial population, atoms have randomly generated coordinates and are placed in the area of the unit cell with periodic boundaries. Dimensions, the rectangular or triclinic type of the unit cell, as well as the number of atoms, are part of a set of parameters of the simulation. Such an approach allows to control the value of atomic density of the newly-created structure. The periodicity of the atomic structure significantly reduces the number of design variables.

The fitness function is formulated as the total potential energy of the considered atomic system, i.e., the total sum of all potential energies of particular atomic interactions. The AIREBO potential in the following form is used in computation:

$$
F F=\sum_{i} \sum_{j \neq i}\left(E_{i j}^{R E B O}+E_{i j}^{L J}+\sum_{k \neq i, j} \sum_{l \neq i, j, k} E_{k i j l}^{T O R S I O N}\right)
$$

where: $E^{R E B O}$ corresponds to the short range interactions between covalently bonded pair of atoms, $E^{L J}$ is responsible for the long range interactions and is computed in a simplified way, using the Lennard-Jones-like function with additional distance-dependent switching functions and $E^{\text {TORSIONAL }}$ is torsional potential which depends on the neighboring atom's dihedral angles.

In order to avoid the situation when distances between atoms are very small, the initial and offspring populations have to be equilibrated, i.e., the potential energy has to be minimized by correction of the positions of atoms. The CG algorithm is used for this purpose. This routine is invoked in each iteration of EA for all individuals in the processed population and temporarily pushes solutions into the local minima.

Such an approach assists in forming of the new, real carbon-based molecular structure, i.e., during the conjugate gradient minimization, each individual - a certain spatial configuration of atoms, starts to form a unique, hybridization-dependent, geometry of flat carbon networks. This step ensures that EA does not process the sets of randomly placed atoms, but operates on fragments of properly bonded carbon structures. Additionally, this method ensures that the optimized structure of atoms is properly equilibrated. The coordinates of atoms are exchanged between EA and the LAMMPS software package and the equilibration process is performed using the minimization method based on the Polak-Ribiere algorithm. The periodicity of the newly-created structure is also achieved in this step by proper boundary conditions, imposed on the unit cell. After the CG minimization of the potential energy, the objective function is computed for each individual in the population. The CG optimization is the most timeconsuming part of the algorithm. To overcome this problem, the authors decided to parallelize the proposed algorithm and make it suitable for running on multiprocessor computers. Thus, the population is scattered into certain number of parts using the MPI library. In the next step, each part is further processed in the parallel way using the dedicated instance of LAMMPS running on a separate core or node of the computer (Figure 1). 


\section{Numerical tests and results}

In order to validate the accuracy of the presented methodology, certain arrangements of carbon atoms already known from literature have been examined, e.g. the supergraphene (triclinic unit cell containing 8 carbon atoms) and the graphyne (triclinic unit cell containing 12 carbon atoms). Since all the tests yield promising results, the proposed optimization algorithm has been applied to search for new stable configurations of a given number of carbon atoms in a unit cell of given size and periodic boundaries (Mrozek et al. 2015). For eight carbon atoms placed in the $4 \AA \times 7 \AA$ rectangular unit cell obtained a stable flat network named X (Figure 1A) and for the same number of carbon atoms placed in the rectangular unit cell $4 \AA \times 6 \AA$ obtained a stable flat network named Y (Figure 1B).

(A)

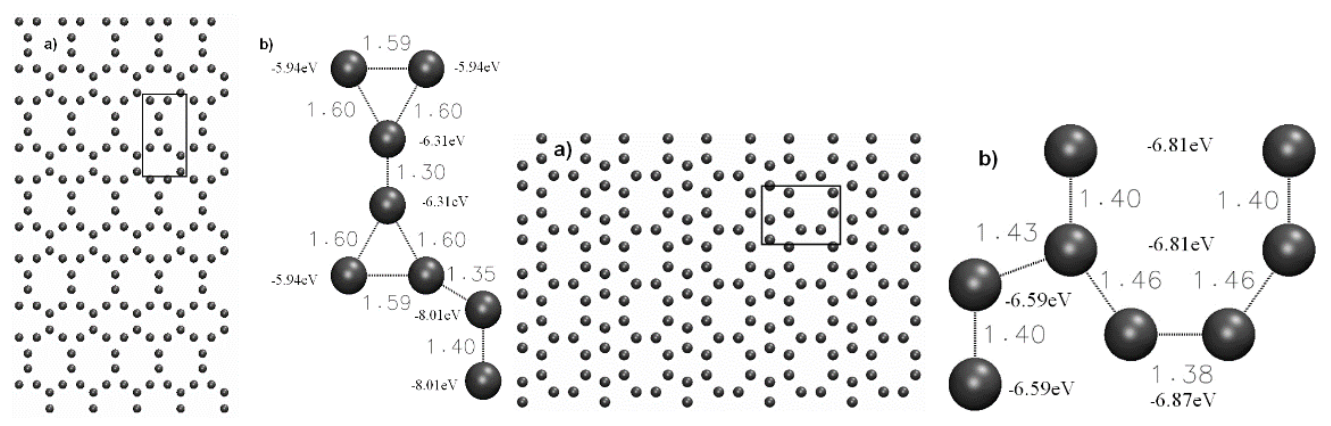

Fig. 1. Layout of new stable carbon networks X (A) and Y (B) found by the hybrid algorithm

\section{Conclusions}

The main purpose of this paper was to present the hybrid parallel algorithm, applied to searching for new 2D graphene-like materials. The proposed method is able to find alreadyknown structures like supergraphene and graphyne as well as new stable ones, named $\mathrm{X}$ and $\mathrm{Y}$. The mechanical properties of the newly-obtained flat carbon networks $\mathrm{X}$ and $\mathrm{Y}$ are also computed. Examples performed for new carbon networks clearly show that the final form and properties of optimized structures depend on the assumed size, type and atomic density of the unit cell. Thus, the considered optimization problem can be reformulated and applied to searching for a molecular structure with predefined material properties, not only in the case of carbon-based structures.

Acknowledgements The authors acknowledge support of the NCN Poland, grant UMO2016/21/B/ST8/02450.

\section{References}

Mrozek A, Kuś W, Burczyński T (2015). Nano level optimization of graphene allotropes by means of hybrid parallel evolutionary algorithm, Computer Material Science 106: 161-1.

Stuart SJ, Tutein JA, Harsison A (2000). A reactive potential for hydrocarbons with intermolecular interactions. J. Chemical Physics 112: 6472-6486. 